\title{
Construyendo el territorio tras Caseros: los jueces de paz y la administración pública en la formación del Estado de Buenos Aires
}

\author{
Constructing territory after Caseros: jueces de paz and public \\ administration in the formation of the Buenos Aires State
}

\author{
Mariana Canedo*
}

\section{RESUMEN}

La construcción del territorio en tanto elemento constitutivo de los Estados fue un proceso más dinámico y complejo de lo que se suele considerar. En el marco de la formación del Estado de Buenos Aires, separado de la Confederación Argentina, el Departamento Topográfico impulsó un proceso de búsqueda de información sobre los límites de las jurisdicciones internas (partidos) que fue llevado a cabo por las autoridades locales (jueces de paz) hacia 1854. El objetivo del artículo consiste en analizar criterios utilizados por las autoridades locales para establecer los límites de sus jurisdicciones, y cuáles fueron los ajustes propuestos en su territorio. También, se consideran las características de la comunicación y utilización de información entre la administración tanto a nivel del gobierno provincial y las autoridades locales, como entre las propias reparticiones del Estado. Argumentamos que los procesos de gestión del territorio en una coyuntura compleja de convulsión política y de limitaciones en la propia administración tuvieron una concreción dilatada. Los procesos de articulación para consensuar límites jurisdiccionales que permitiesen un mejor control del territorio y la población implicaron hacia mediados del siglo XIX, la aceptación de distintas relaciones sociales y políticas por parte de las autoridades locales y provinciales.

Palabras Claves: Estado de Buenos Aires. Territorio. Juez de paz. Departamento topográfico. Mesa estadística.

\section{ABSTRACT}

The construction of territory as a constitutive element of States was a much more dynamic and complex process than it is made out to be. Within the framework of the Buenos Aires State formation, separated from the Argentine Confederation, the Topographical Department prompted a process of information search on the limits of internal jurisdictions (partidos) that was carried out by local authorities (jueces de paz) towards 1854. The aim of this articule is to analyze the criteria used by local authorities to establish limits to their own jurisdictions, and the adjustments proposed within their territory. In addition, the nature of information use and communication within the administration is taken into consideration, both on a provincial and local level as well as in the State's own distribution. We argue that territorial management processes in a complex situation of political turmoil and inherent administrative limitations had a prolonged realization. Articulation processes aimed to reach a consensus on jurisdictional limits that would allow an improved control of both territory and population entailed, towards the mid $19^{\text {th }}$ century, the acceptance of different social and political relations by local and provincial authorities.

KEYWORDS: Buenos Aires State. Territory. J ustice of the peace. Topographical department. Statistical Office.

\footnotetext{
* Dra. en Historia, Profesora e Investigadora de la Universidad Nacional de Mar del Plata y el CONICET, República Argentina
} 
[...] ni los mismos J ueces de Paz pueden saber de un modo positivo si proceden o no dentro del límite de su jurisdicción, ni nadie puede expedirse en este asunto con certeza, de donde se originan graves entorpecimientos. Los particulares ocurren a esclarecer sus dudas a este Departamento [Topográfico, MC], y aun de los Ministerios mismos, se han pedido varias veces Cartas topográficas donde se hallen demarcados los limites de los Partidos, pero el Departamento carece absolutamente de datos ciertos, ni aun de lo poco que se ha estatuido por la Superioridad tiene conocimiento alguno"2

En noviembre de 1853, los ingenieros a cargo del Departamento Topográfico de Buenos Aires, llamaron la atención al Ministro de Gobierno sobre los inconvenientes generados a particulares y autoridades por la incertidumbre con que se hallaban establecidas las divisiones jurisdiccionales internas de la provincia. La nota era directa en expresar la carencia de información actualizada por parte del propio Departamento y proponía obtenerla recurriendo a los jueces de paz, autoridades de las jurisdicciones locales. ${ }^{3}$

Los jueces de paz debían presentar una "Relación” (informe) de los limites de sus respectivos partidos "según los ha establecido la práctica, teniendo en vista las poblaciones, los terrenos, los arroyos o cualquiera otro punto notable”. ${ }^{4}$ La información podía ir acompañada de las observaciones que las autoridades locales juzgaran oportunas para que "su autoridad sea ejercida directamente sobre los habitantes de su jurisdicción” Una vez reunida la información y confrontados los datos, el Departamento Topográfico se proponía realizar de "modo fijo" una demarcación de límites sobre una nueva carta topográfica de Buenos Aires. A los dos días de enviada la nota, el ministro Irineo Portela manifestó el aprecio del gobierno por "el loable empeño" ante el tema propuesto. 5

La propuesta de los ingenieros del Departamento Topográfico fue una iniciativa específica que, junto a otras, forma parte del proceso de construcción del territorio que fue compartido por diferentes formaciones estatales de América y otros continentes durante el siglo XIX. Abundante bibliografía ha señalado que este proceso fue, en conjunto, complejo, conflictivo, discontinuo y no lineal, y que tuvo variadas manifestaciones y dinámicas regionales. Para el caso de Buenos Aires, la construcción del territorio durante el siglo XIX se vinculó con las cambiantes fronteras poblacional,

\footnotetext{
2 Archivo General de la Nación (en adelante, AGN), Sala X, Ministerio de Gobierno del Estado de Buenos Aires (en adelante MGEBA), legajo 5290. Nota de Saturnino Salas al Ministro de Gobierno Irineo Portela, 14 de noviembre de 1853. Los partidos eran jurisdicciones internas de una gobernación o, después de 1820, de una provincia.

3 Ante la abundante bibliografía sobre los jueces de paz, remitimos a modo de ejemplo que caracteriza una mirada en conjunto de estas autoridades e incluye un período amplio de su funcionamiento, a GELMAN 2000.

${ }^{4}$ Los resaltados en itálica son nuestros.

5 AGN, Sala X, MGEBA, legajo 5290
} 
agraria, mercantil, de ocupación y apropiación de la tierra, con parcialidades indígenas, pero también una frontera política y bélica que evidenciaba las luchas de poder en los distintos proyectos de organización provincial y de país.

La propuesta del Departamento Topográfico de Buenos Aires sobre la delimitación de las jurisdicciones internas se generó en una coyuntura particular. En 1852, tras la batalla de Caseros y la consecuente derrota del Gobernador de Buenos Aires Juan Manuel de Rosas, una serie de nuevos enfrentamientos entre la Confederación Argentina y la provincia de Buenos Aires pusieron de manifiesto tenaces tensiones existentes en torno al rumbo a tomar por la provincia. (HALPERIN DONGHI, 1995; LETTIERI, 2006; BONAUDO, 2007) Los conflictos devinieron en la separación de Buenos Aires, pero también en un contexto bélico y de convulsión política que impregnó, por varios años, la vida de sus habitantes y las decisiones políticas y económicas de los gobiernos. Por otro lado, la conformación del Estado de Buenos Aires generó un escenario político estimulante (sin dejar de ser conflictivo) para modelar e intervenir en un nuevo proyecto de construcción de un Estado, siendo estos primeros años de elaboración en el plano jurídico, institucional, y político (elaboración y sanción de la Constitución del Estado de Buenos Aires, de la Ley de Municipalidades, de organización administrativa-institucional, debates antecediendo la sanción de los Códigos). Por último, de manera más específica, no podemos dejar de señalar la evolución del aumento de los precios de la tierra a partir de 1820, tras una larga estabilidad durante el período colonial, ocupando la tierra un lugar secundario entre los medios de producción (FRADKIN; GARAVAGLIA, 2004) y los debates iniciados tras Caseros sobre la tierra pública (VALENCIA, 2005).

En este contexto, durante el año 1852 se había impulsado desde los cambiantes gobiernos de Buenos Aires el "restablecimiento" del Departamento Topográfico, una institución conformada en la década de 1820 y nuevamente valorada por sus contemporáneos como "la más necesaria" y que buscaba instituirse como modelo para "la República”. Sin embargo, aunque paradigmático, no fue este el único esfuerzo realizado desde los gobiernos de Buenos Aires para jerarquizar el desarrollo institucional del Estado, por lo que se generaron o actualizaron otras reparticiones como el Departamento de Obras Públicas o la Mesa Estadística. Esta situación en un contexto de relativa escasez de recursos y de personas formadas para emprender una simultaneidad de requerimientos puso en evidencia limitaciones y discrepancias en la propia administración.

La construcción de cartas topográficas o catastros fue un proceso que llevó a la apropiación simbólica del territorio por parte de las instituciones públicas, como señala 
J uan Pro "refleja una visión del Estado", señalando la importancia de investigar los medios sustitutivos del catastro propiamente dicho, que se usaron para gestionar la relación entre el territorio, la sociedad y el Estado en formación. Recientemente, Gautreau y Garavaglia han profundizado el estudio de las relaciones entre el Departamento Topográfico de Buenos Aires y los agrimensores como punto clave para comprender las limitaciones de la administración para obtener información sobre el territorio a nivel local. (PRO, 2011; GAUTREAU, GARAVAGLIA, 2011).

La nueva carta topográfica sobre Buenos Aires con los límites "fijos" de los partidos propuesta por el Departamento Topográfico en 1853 no se concretó por esos años, sino una década después. Sin embargo, la documentación generada durante el año 1854 permite conocer algunos aspectos de la intervención de los jueces de paz, propietarios y otros actores locales en las relaciones sociales de poder que significó la construcción del territorio. Las más de 40 jurisdicciones locales sobre las que ha quedado algún tipo de documentación ponen de relieve, una vez más, la dinámica heterogeneidad de Buenos Aires

En el marco de diferentes intereses en torno a la construcción del territorio en la formación del Estado de Buenos Aires, nos proponemos analizar el proceso de obtención y circulación de la información sobre los límites de las jurisdicciones internas, considerando el accionar de diferentes actores locales. ¿Cuáles fueron los criterios que primaron en el establecimiento de los límites de los partidos? ¿Cómo se articularon los poderes locales con los integrantes del Departamento Topográfico para precisarlos? ¿Cómo se gestionó el proceso de obtención y comunicación de la información sobre las jurisdicciones por parte de las autoridades locales y de las instituciones del Estado del Buenos Aires? ¿Qué relaciones de poder se pusieron en juego en la delimitación de los límites?

Los procesos de construcción del territorio en una coyuntura compleja de convulsión política y de limitaciones en la propia administración estatal tuvieron una concreción dilatada. Consideramos que los procesos para consensuar límites jurisdiccionales que permitiesen un mejor control del territorio y la población implicaron, hacia mediados del siglo XIX, la consolidación de las relaciones de apropiación de la tierra por particulares, y políticas por parte de los poderes locales y las autoridades del Estado de Buenos Aires. ${ }^{6}$

\footnotetext{
${ }^{6}$ Las fuentes utilizadas son fundamentalmente las notificaciones oficiales entre el Presidente del Departamento Topográfico y el Ministro de Gobierno, los informes presentados por los jueces de paz a ambos, el Registro Estadístico del Estado de Buenos Aires, y las notificaciones del J efe de la Mesa Estadística al Departamento Topográfico. La documentación se encuentra dispersa en los legajos del Ministerio de Gobierno del Estado de Buenos Aires y en los sobres de "Documentos
} 


\section{La Construcción de una Nueva Carta Topográfica para Buenos Aires}

El conocimiento del territorio como instrumento para un mejor accionar de los gobiernos no surgió como objetivo en Buenos Aires por primera vez a mediados del siglo XIX. Tampoco el requerimiento de una carta topográfica con este fin. Sin embargo, una nueva combinación de situaciones permitió actualizar esta iniciativa que fue resuelta solo en la medida en que se alcanzó la suficiente acumulación de información con la calidad adecuada. Precisaremos algunas situaciones al respecto.

A mediados del siglo XIX, el proceso político transcurrido desde la etapa de creación institucional en la década de 1820, había alejado al Departamento Topográfico de su vinculación casi fundacional con la enfiteusis impulsada por Bernardino Rivadavia. ${ }^{7}$ Después de la batalla de Caseros, la enfiteusis quedó subsumida como problemática en los debates jurídicos y políticos sobre la situación de las tierras públicas en la provincia (VALENCIA, 2005; ZEBERIO, 2009). Para el Departamento Topográfico "restablecido" desde 1852, los proyectos más ambiciosos se centraron en el "arreglo" de los pueblos de la campaña, la realización del plano de la ciudad de Buenos Aires, y la elaboración de la Carta Topográfica de la provincia o Registro Gráfico (CANEDO, 2011) Las tres propuestas evidenciaban, en distintas escalas, la intención sobre el control del territorio por parte del Estado de Buenos Aires.

Cabe precisar que el propósito de contar con una carta topográfica de la provincia como instrumento que otorgaba a los gobiernos información para actuar, antecedió al Departamento Topográfico de Buenos Aires pese a su carácter de pionero dentro de las instituciones topográficas en Sudamérica. En 1812, Miguel de Azcuénaga, ocupando el cargo de gobernador intendente creado por el Triunvirato, decretó se levantara un plano topográfico de la provincia. ${ }^{8}$ (ZINNY, 1987, p.179; ESTEBAN, 1962, p. 12).

Al conformar el gobierno de Buenos Aires, una "Comisión Topográfica” en septiembre de 1824, se estableció en sus primeros artículos que quedaba "encargada de

antiguos" de cada partido del Archivo Histórico de Geodesia del Ministerio de Obras Pública de La Plata. También utilizamos a Maeso (1855).

7 Cabe recordar que en el gobierno de Martín Rodríguez (1820-1824) se comenzó a utilizar las tierras públicas como garantía de la deuda de la provincia, prohibiéndose la venta de las mismas desde 1822. El gobierno entregó a particulares el uso de la tierra bajo contrato enfitéutico. Entre las fundamentaciones a la constitución del Departamento General de Topografía y Estadística, se explicitaba el "deber que tiene el Gobierno de preparar los medios para la ejecución de la ley que manda dar en enfiteusis las tierras de propiedad publica, la necesidad de empezar y arreglar la topografía y estadística del país, [...]" Al día siguiente del decreto, en junio de 1826, el gobierno de Rivadavia reglamentó la ley sobre tierras en enfiteusis que había sido aprobada un mes atrás (BUENOS AIRES, 1947, p.3-4).

8 El objetivo del plano se planteaba en términos de poder "repartir gratuitamente a los hijos del país suertes de estancias proporcionadas y chacras para la siembra de granos bajo un sistema político que asegura el establecimiento de poblaciones y la felicidad de tantas familias patricias que siendo victimas de la codicia de los poderosos, viven en la indigencia, y en el abandono con escándalo de la razón y perjuicio de los verdaderos intereses del Estado." (ESTEBAN, 1962, p. 13). 
reunir los datos para la formación del plano topográfico de la Provincia” (art. 3o) La decisión quedó reforzada con la ley sancionada en noviembre por la Sala de Representantes de la Provincia, autorizando al gobierno "a ejecutar la mensura general de las tierras de la Provincia y levantar el plano topográfico correspondiente". (BUENOS AIRES, 1947, p. 7-8). La comisión, tras un año y medio de funcionamiento, presentó una "Memoria" al entonces presidente Bernardino Rivadavia donde explicitaba las tareas realizadas, señalando dificultades vinculadas con la calidad de los datos para emprender la carta topográfica: "útiles [...] a otros objetos, ellos (los datos, $\mathrm{MC})$ dejaban sin embargo un alto grado de incertidumbre en la deliberaciones de la Comisión". ${ }^{9}$ Se adelantaba en la "Memoria" que la comisión se encontraban realizando un nuevo plano "trabajado bajo nuevos datos", con escala de media pulgada por legua, aclarando que:

En él se dejan en blanco las partes que aun no están conocidas, y esta carta que no es más que un simple croquis, tiene ya un grado de certeza capaz de guiar a la Comisión en sus informes, y ser útil a los diferentes objetos del ministerio. Ella está apoyada en mensuras que la Comisión ha examinado, en extractos de otras que han sido practicadas anteriormente y en los viajes que ha hecho el Señor D. Felipe Senillosa miembro de esta Comisión. 10

Era claramente un proceso que llevaba tiempo y esfuerzo sostenido para acumular información adecuada. La modalidad elegida, como señalan Gautreau y Garavaglia, se basaba en el plano individual ("individual-map-based cadastre") y el conocimiento del Estado sobre su territorio dependía de la cantidad de planos que se lograban en cada periodo (GAUTREAU, GARAVAGLIA, 2011). Para ese año de 1826 la Comisión proyectaba "adelantar la parte del Norte que se halla aun atrasada por la falta de datos" e incorporar la traza de una meridiana y una perpendicular, que a manera de ejes facilitaran el establecimiento de mojones guías. En el informe, Vicente López, Felipe Senillosa y Avelino Díaz, los integrantes de la comisión que acompañarán desde distintos cargos la actividad topográfica en Buenos Aires por décadas, afirmaban:

Por este medio el territorio de la Provincia de Buenos Aires en la oportunidad que presenta la regularidad de su suelo, ofrecerá el primer modelo de una carta permanente, y un ejemplo de división territorial en el que están a la vez ligados el interés privado, el del pueblo y el progreso delas ciencias. ${ }^{11}$

\footnotetext{
${ }^{9}$ Archivo Histórico de la Provincia de Buenos Aires (en adelante AHPBA), Departamento Topográfico de la provincia de Buenos Aires (1821-1865), C-49; A.2, leg. 1.

${ }^{10}$ AHPBA, Departamento Topográfico..., C-49; A.2, leg. 1.

11 AHPBA, Departamento Topográfico..., C-49; A.2, leg. 1.
} 
Será en 1833 cuando el Departamento General de Topografía y Estadística publique el Registro Gráfico de la Provincia de Buenos Aires, construido en base a las mensuras realizadas de terrenos de particulares. ${ }^{12}$ Saturnino Salas, quien había participado en el primer Registro como integrante del Departamento Topográfico, sentenciaba tres décadas después que la primera carta topográfica "por falta de conocimientos había sido bastante inexacta y deficiente".

El segundo Registro Gráfico se publicó en 1865. Incorporaba la información acumulada por el Departamento en estas décadas que habían sido las de mayor producción de mensuras, e incluía como base al Archivo del Departamento. ${ }^{13}$ Sumaba también parte de los datos presentados por los jueces de paz sobre los límites de los partidos.

En 1863, Salas como presidente del Departamento le escribió al Ministro de Gobierno de entonces, Mariano Acosta:

Mucho tiempo ha que el Departamento Topográfico trabaja con asiduidad en la construcción del Plano General de la Provincia o sea el Registro Gráfico de las propiedades rurales, con el designio de la Administración y el Público reportarán de él. Aun no ha sido posible terminarlo del todo, pero lo estará sin duda en pocos meses más [...]

El Registro va a dar conocimientos exactos de la subdivisión del territorio de la Provincia; del modo como están ubicadas las propiedades con su verdadera extensión y todos los detalles topográficos que el Departamento ha podido adquirir desde su creación. Servirá, Señor, para poder determinar una nueva división de Partidos, obra reclamada urgentemente y que sin el plano de la Provincia es imposible llevar a cabo."14

El objetivo de la nota de Salas era ir adelantando el financiamiento del grabado e impresión de registro. Consideraba que si se imprimía en el país, tendría menos errores que si se hiciera en Europa, como se había pensado y se había realizado con el registro de 1834. Sobre la base de una primera edición, que se había agotado, se había publicado otra en Londres por Mr. J ohn Arrowsmith, "en escala menor que con todos sus errores y falta de detalles, se ha vendido siempre a muy alto precio relativamente en esta Ciudad".

12 AGN, Sala X, MGEBA, legajo 22957. Para un análisis de los alcances de información de los registros gráficos, remitimos a la tesis doctorado de G. Banzato (2002) y a las Figuras 1, 11, 12a y 12b en GAUTREAU; BOULLIER; CUÉNOT ( 2011).

13 Sobre la valoración del Archivo del Departamento Topográfico hacia mediados del siglo XIX se ha señalado: "El archivado, mucho más que el mapeo, fue el invento del momento" El propio Salas hacía referencias sobre el Archivo del Departamento como "único en su tipo". Esta capacidad otorgó al Departamento mayor autonomía con relación a los agrimensores. (GAUTREAU, GARAVAGLIA, 2011, p. 95).

14 AGN, Sala X, MGEBA, legajo 22957. Nota de Saturnino Salas a Mariano Acosta, 25 de Septiembre de 1863. 
Adjuntaba tres presupuestos de litógrafos de Buenos Aires comparando calidad y precio. El financiamiento se realizaría por el Ministerio de Gobierno, en base suscripciones que el Departamento comenzaría a publicitar. Para cuando hubiesen cubierto los gastos, Salas ya anunciaba que con las utilidades de los 3000 ejemplares, podrían afrontar "otros trabajos igualmente importantes, tales como la conclusión, reducción y publicación del Plano de la Ciudad; que no tiene exacto y que es igualmente necesario". ${ }^{15}$

\section{La Obtención de Información Sobre los Partidos}

En abril de 1854, a seis meses de haberle presentado al ministro Portela la propuesta de requerir información a los jueces de paz sobre los limites de sus propias jurisdicciones, Salas manifestaba que no podía dar comienzo a los trabajos de demarcación de partidos que se había propuesto trazar en la Carta topográfica de la Provincia porque "no han contestado todos los Jueces de Paz, como era de esperar" . ${ }^{16}$ La "loable" tarea propuesta, además de requerida por particulares y el gobierno, tuvo como característica la dispersión de informantes y morosidad en las respuestas. Los jueces de paz fueron enviando información sobre sus jurisdicciones entre diciembre de 1853 y agosto de 1854, pese a las insistencias del presidente del Departamento.

En nota dirigida al Oficial Mayor del Ministro de Gobierno, Don J osé M. la Fuente, Salas señaló -uno por uno- a los 31 jueces de paz que no habían contestado oportunamente. ${ }^{17}$ Como respuesta, el gobierno autorizaba al Departamento Topográfico a dirigirse a los jueces de paz de manera directa, pero específicamente sobre el tema.

En julio, Salas presentó una actualizada “Relación de los J ueces de Paz que no han mandado los límites de sus partidos." La lista había disminuido de 31 a 10 jueces. ${ }^{18}$ Solicitaba al ministro "se sirva ordenar a los expresados J ueces de Paz remitan a esta oficina lo más pronto posible los datos anteriormente solicitados, [...]" En sus respuestas, varios jueces de paz hacen alguna referencia al "tenor" de las notas. ${ }^{19}$ Entre las justificaciones por la demora en la respuesta a lo solicitado se encuentran la ausencia de conocimiento sobre tema por haber asumido recientemente o por no haber recibido la comunicación. También se hicieron referencias a haber dirigido ya la

\footnotetext{
15 AGN, Sala X, MGEBA, legajo 22957

16 AGN, Sala X, MGEBA, legajo 5290

17 AGN, Sala X, MGEBA, legajo 5290

18 AGN, Sala X, MGEBA, legajo 7648

19 AGN, Sala X, MGEBA, legajo 7648 El J uez de Paz de la Villa de Luján respondió a los pocos días "y en contestación dice que ha dado cumplimiento a lo ordenado" (tachado decía "pedido") ;
} 
respuesta directamente al Ministro de Gobierno o parte de la información al J efe de Policía. ${ }^{20}$

El Juez de Paz de Navarro le escribe a Salas diciendo que "se permite informar a $\mathrm{V}$ que en el mes de Febrero anterior se pasó al ministerio de gobierno la demarcación de los limites de este Partido, en conformidad a la citada Superior resolución" El J uez de Paz de Bragado cuando "pasa a exponer los limites que por la práctica y según la voz del vulgo dividen este Partido", aclaraba que los "conocimientos que el que firma puede transmitir en cuanto a Población, superficie del territorio y demás; coexisten en la Estadística que el que firma remitió al Sr J efe de Policía”21

Por su parte, el Juez de Paz de San Nicolás, el partido con mayor densidad poblacional de Buenos Aires ubicado en el extremo norte de la provincia, en plena zona de pasaje de tropas de la Confederación tanto como del Estado de Buenos Aires, expresaba su situación en estos términos:

[...] que en la actividad me estoy ocupando en la Mayor Constancia a fin de remitir a la mayor brevedad la Estadística de todo el partido y en él hice también comprendida la extensión y límites del distrito. Si ha habido retraso, Sr. Ministro, en la remisión de tales noticias topográficas, es a causa precisamente del mucho recargo concurriendo también la falta de personas que ayuda en estos trabajos." 22

A distintos ritmos, las respuestas de los J ueces de Paz sobre sus jurisdicciones fueron llegando en el plazo de unos nueve meses, con un rango de información variada, y explicitando distintas formas en que las propias autoridades locales accedieron a la información de los límites. El Juez de Paz de San Antonio de Areco, uno de los primeros poblados y jurisdicciones establecidos en la campaña, no solo respondió prontamente, sino que explicitó la forma de acceso a la información y formuló una nueva propuesta de límites:

20 "Persuadido el infrascrito por el tenor de la nota de Us que tal vez ignore que en la fecha de la circular, 16 de noviembre del año pasado a que se refiere, no estaba el que firma al cargo del J uzgado de Paz de este Partido [Cañuelas, MC] y que en el mes de Febrero del presente año, que tomó posesión ningún conocimiento le fue transmitido sobre este asunto y por consiguiente le era del todo desconocida la enunciada circular." AHGyC, Documentos antiguos, Partido de Cañuelas, nota del 27 de mayo de 1854. En el mismo sentido escribió el J uez de Paz de San Fernando, “debió recibirse mucho antes de estar a cargo este Juzgado; sin embargo debe estar seguro el Señor Presidente que a la mayor brevedad le serán transmitidos los datos que pide." AHGyC, Documentos antiguos, Partido de San Fernando, nota del 23 de mayo de 1854

21 AHGyC, Documentos antiguos, Partido de Navarro, nota del 3 de agosto de 1854. AHGyC, Documentos antiguos, Partido de Bragado, nota del 10 de junio de 1854

22 AGN, Sala X, MGEBA, legajo 7648 
[...] reunió los vecinos mas antiguos de este Partido, e igualmente algunos otros vecinos linderos de él, y procedimos a formar una nueva demarcación de limites para este Partido, la que tengo el honor de acompañar: como también los limites actuales de el que no son conocidos, [...] como VS se impondrá por la planilla que acompaño. También he creído necesario para mayor conocimiento de los caminos y población de este Partido remitir al $\mathrm{V}$, un padrón o ligera estadística de todo el."23

Algunos Jueces de Paz recorrieron la zona para responder con mayor precisión, comisionaron a un vecino del pueblo (y le brindaron medios para hacerlo), o generaron una "comisión auxiliadora" encargada de realizarlo:

[...] para cuyo efecto [el Juez de Paz, MC] ha recorrido y examinado toda la área que corresponde al Partido, y acompaña un Croquis y cuadro Estadístico"24

[...] remite a $\mathrm{V}$ la nota y plano que le ha presentado el vecino comisionado por este Juzgado para aquel efecto Dn Nicolás González" AHGyC, Documentos antiguos, Villa de Mercedes, nota del 7 de julio de 1854.

La ubicación del partido de Patagones, en el extremo sur de Buenos Aires, en zona de frontera con las parcialidades indígenas otorga singularidad a la situación, y el Juez de Paz participa a vecinos, al Comandante y coordina objetivos en el esfuerzo realizado para establecer los límites:

\begin{abstract}
Al efecto reunió la Comisión Auxiliara de este J uzgado para ilustrar el juicio del infrascrito, y además hizo comparecer en este J uzgado a los mas antiguos vecinos de este Pueblo, [...]. Posteriormente y para poder cerciorarse mejor partió el que suscribe acompañado del Sor Comandante del Partido, Alcalde y muchos vecinos hasta una distancia de 22 leguas inspeccionando por si mismo lo mas prominente que se presentaba a su vista en su ida y regreso. Influía también mucho en esta expedición el deseo de encontrar un paraje adecuado para establecer una Guardia de Fuerza que se espera de la Capital para ser una segura manzana para la protección de la Frontera de estos Establecimientos agrícolas y pastoriles. ${ }^{25}$
\end{abstract}

A su vez, el juez de Paz de Federación, presentó la información básica sobre los límites: “No habiéndose recibido en esteJ uzgado la nota a que se refiere el Sor Ministro

\footnotetext{
23 AHGyC, Documentos antiguos, Partido de San Antonio de Areco, nota del 30 de diciembre de 1853.

24 AHGyC, Documentos antiguos, Partido de La Matanza, nota del 8 de diciembre de 1853.

25 AHGyC, Documentos antiguos, Partido de Patagones, nota del 30 de marzo de 1854.
} 
ignora la forma en que se me pide dicho conocimiento por lo que me limito a decir que este partido se divide al norte con el de Rojas [...]"26

Mientras que el Juez de Paz de Mar Chiquita, ubicado en una zona de más ocupación, expresó sus limitaciones para cumplir el pedido:

Es imposible dar un conocimiento exacto de los limites que abraza este Partido, como V me lo indica en su nota a consecuencia de no contar en este Partido, persona alguna capaz de ese desempeño, pues todos están en el mismo caso en que yo me hayo respecto a la expresada demarcación de los limites de este Partido: y a mas ser imposible el formar una comisión para ese efecto a lo que siente el que suscribe no poder cumplir con un asunto de tanto interés publico".

Por último, consideremos al observación realizada a Salas por parte del J uez de Paz y Comisario de la Villa de Lujan, alertando sobre la calidad de la información que presentaba:

Se han podido recabar por tradición y noticia de algunos vecinos antiguos. V debe convencerse que estos no pueden ser con la exactitud necesaria para levantar la carta Topográfica, que solo serán aproximadamente, pues este Partido jamás ha sido deslindado científicamente y por consiguiente no se conocen con exactitud sus límites. Sin embargo diré en aquel sentido lo que se sabe."27

La calidad de información recibida por el Departamento Topográfico, como observaba el J uez de Paz de la Villa de Luján, y la consolidación de la misma dentro del propio Departamento, fue una cuestión que acompaño el proceso de construcción del Registro Gráfico, pero también a la actuación de la institución en general. El tema tuvo varias aristas que generaron diferentes posturas y tensiones fundamentalmente dentro de la administración pública. Uno de los ejemplos conocidos se vincula con cuál debía ser el grado de autonomía de la actuación de los agrimensores al confeccionar una mensura y, como contraparte, cuál el nivel de regulación del Departamento sobre el trabajo realizado. Otro, que presentamos a continuación, se refiere a la circulación y consolidación de la información dentro y fuera de la administración, justamente a partir de los informes elaborados por los jueces de paz.

${ }^{26}$ AHGyC, Documentos antiguos, Partido de Federación, 10 de junio de 1854. Similar es la respuesta del Juez de Paz de Monte Caseros: "[...] no han sido remitidos por este juzgado hasta ahora a causa de las muchas atenciones y recargo de este J uzgado y aun por el poco conocimiento que tenia el que firma sobre el particular, pero habiéndolo todo resulta que sus extensión se compone de cuarenta y dos leguas,[...]" AHGyC, Documentos antiguos, Partido de Monte Caseros, 5 de julio de 1854

27 AHGyC, Documentos antiguos, Partido de Luján, nota del 12 de junio de 1854. 


\section{La Circulación y Difusión de la Información por la Administración}

Mientras las notificaciones entre Saturnino Salas, el Ministro de Gobierno y los jueces de paz transcurrían, Juan Bernabé y Madero, "J efe" de la recientemente instalada Mesa de Estadística General de la Provincia Buenos Aires se dirigía también a Salas para obtener información. En marzo de 1854, le informaba al Presidente del Departamento Topográfico que en "uso de las atribuciones" pedía se remitiese "por Trimestre el movimiento de ese Departamento, principiando por mandar en los primeros días de Abril, todos los trabajos que en ese Establecimiento se hayan hecho desde el principio de este año hasta fines del mes que corre." Y detallaba los tópicos sobre los cuales el Departamento debería poder enviarle información. ${ }^{28}$

La conformación de la Mesa Estadística era un nuevo esfuerzo institucional del Estado de Buenos Aires, como lo había sido el restablecimiento del Departamento Topográfico o la conformación del Departamento de Obras Publicas. La Mesa Estadística se ubicaba también como continuación operativa y simbólica de la experiencia rivadaviana, en la que se había destacado como producto el Registro Estadístico de la Provincia de Buenos Aires (1822-1827). La nota de Juan de Bernabé y Madero forma parte de la gestión realizada para retomar la segunda época del mismo, que iniciada en 1854 llegará a 1880, y requería de una fluida comunicación interinstitucional para la obtención de información. ${ }^{29}$ No sería fácil lograrlo, por lo menos, en los primeros años.

Las solicitudes de la Mesa Estadística hacia el Departamento Topográfico continuaron. A los tres meses, una nueva nota recordaba que se estaba por "principiar el 2ํㅡㄹ Registro Estadístico" y se solicitaba “tenga a bien remitirnos algo de lo que ya se

28 La comunicación y el pedido continuaban: "Además espera el que suscribe, que en vista del gran número de edificios que se han levantado y refaccionado desde la feliz terminación de la última guerra civil, pasara V el número de licencias expedidas para unos y otros, desde el 14 de Julio. Además del movimiento por Trimestres que debe $\mathrm{V}$ pasar a esta, acompañará todas las noticias referentes en la Topografía de la Provincia tanto en lo terrestre como en la hidráulica, que se tengan de estos últimos años y que se vayan teniendo como la latitud y longitud de los pueblos y puntos principales, en su situación y extensión, las distancias de estos a la Capital y de ellos entre sí, la extensión de la Provincia en la que tiene poblado, su configuración y confines, sus ríos, lagos, costas de mar, puestos y terrenos anegadizos. Todos los cuales datos no exijo a V por lo pronto sino como dije antes, conforme se vayan teniendo; recomendando a $\mathrm{V}$ por ahora la remisión de los que se tuviesen y pudieran darse." AHGyC, Documentos Antiguos, Departamento Topográfico, Carpeta 38, nota de 8 de marzo de 1854

29 Hernán Otero especifica que los datos para el Registro Estadístico provenían de una amplia red de capilaridades institucionales que incluía, entre otros, el Departamento Topográfico, la Administración de aduanas, los jueces de paz de la campaña, los cónsules extranjeros residentes en la ciudad, y para la información específicamente demográfica, los curas párrocos. El producto fueron 36 volúmenes del Registro Estadístico de la Provincia de Buenos Aires (segunda época) (OTERO, 2006, p.179) 
ha pedido" para la sección correspondiente. El jefe de la Mesa Estadística era directo en su pedido y agregaba:

Cuando todas las autoridades se han apresurado a llenar con agrado nuestros pedidos, como lo vera $\mathrm{V}$ en la impresión que actualmente se hace. Creo, digo, que el Departamento Topográfico no se hará por más tiempo sordo a tantas exigencias y sacrificara un poco de tiempo con el objeto de llenarlos aunque más no sea en parte. El que suscribe así lo espera, no solo por lo que mas antes aduce, si no también por el Patriotismo del Jefe de aquel Depto. y por sus luces que no deben desconocer lo útil que son esos trabajos en la Época que atravesamos. ${ }^{30}$

La respuesta de Salas resulta interesante, ya que permite considerar las diferentes posiciones ante la información demandada ("datos estadísticos" en las referencias de Salas), que habrían fundamentado la dilación con la información que, por otro lado él mismo se encontraba demandando a los jueces de paz. ${ }^{31}$ Salas comenzó aclarando que adjuntaba "los datos estadísticos" que el Departamento ("esta oficina") había podido suministrar para el trimestre mayo-junio, y que anteriormente había enviado para enero-abril. Especificaba que los datos sobre la situación geográfica de los pueblos, que habían figurado en una tabla en el Registro en el primer trimestre, no iba a ser actualizada por no tener datos nuevos, pero además argumentaba que:

Y el que suscribe añadirá que esos mismos datos que le fueron remitidos no merecen a su juicio la fe suficiente para figurar como de base de calculo geográfico ninguno pues ellos han sido tomados de apuntes aislados que existen en los archivos de esta oficina. No se han hecho hasta hoy en el país trabajos científicos sustentados con este objeto, y por consiguiente no hay datos que puedan considerarse como exactos." ${ }^{\prime 32}$

En diciembre de 1854, una nueva nota del J efe de la Mesa Estadística solicita "a la brevedad" a Salas información justamente sobre los partidos de la campaña (cuáles eran, su agrupación por zonas, la distancia de la capital, etc.) "para

30 AHGyC, Documentos Antiguos, Departamento Topográfico, Carpeta 38, nota de 16 de junio de 1854

31 Dos notas del 3 y 4 de julio aparecen como respuesta por parte del Presidente del Departamento Topográfico a los planteos del jefe de la Mesa Estadística. Desconocemos si la primera nota fue efectivamente mandada, ya que se encuentra sin firma y los temas que aborda son retomados al día siguiente con mayor profundidad. Las referencias corresponden a la nota del día 4. AHGyC, Documentos Antiguos, Departamento Topográfico, Carpeta 38, notas del 3 y 4 de julio de 1854

32 Salas precisa aun más: "Lo que suministra el registro gráfico de las mensuras publicada en la parte poblada del Estado, no pueden figurar en el registro estadístico por su falta de exactitud El Departamento Topográfico pues no puede dar como exactos datos que en su conciencia merecen poca o ninguna fe. Durante toda la época del sistema de gobierno anterior a Caseros esta oficina se puede decir que no ha funcionado." Para finalizar, Salas aclaraba que su comportamiento no era "falta de patriotismo" ni falta de valoración sobre "la importancia de una publicación tan útil en de la estadística exacta del país, [...]" 
complementar ciertos trabajos que esta Oficina debe presentar en estos días al Sup.or Gobierno". También aprovechaba para recordarle a Salas "el envío a su tiempo de los trabajos de esa Administración en el Semestre $2^{\mathrm{o}}$ de este año."33

En enero de 1855, el J efe de la Mesa Estadística es remplazado por J usto Maeso, quien concretará la publicación del Registro Estadístico correspondiente al segundo cuatrimestre de 1854. El volumen comienza con una "Advertencia” realizada por Maeso en mayo de 1855:

Antes de concluir, séame permitido deplorar la irregularidad con que las autoridades en general trasmiten los datos é informes á que están obligados por el superior decreto de Diciembre 23 de 1853, que fundó este Departamento de Estadística. Mucho se adelantaría en la importancia de los datos trasmitidos, y en la celeridad y exactitud de su trasmisión, si el Exmo. Gobierno tuviese á bien declarar en vigencia el decreto sobre Razones Estadísticas, de fecha 15 de Marzo de 1822, expedido en el Ministerio de D. Bernardino Rivadavia; ó bien, recomendar públicamente el cumplimiento del mencionado decreto ereccional de esta Oficina [...]

Muy útil seria, pues, que el Exmo. Gobierno advirtiendo estas imperfecciones en el servicio público, tuviera á bien ponerles un eficaz remedio. Jamás podrá arribarse á la posesión de grandes conocimientos revelados por la Estadística, sino se adopta un sistema coercitivo adecuado al caso; y desde que el Exmo. Gobierno está persuadido de la importancia de las demostraciones estadísticas ¿por qué no adoptarlo sin demora?"34

La crítica generalizada de Maeso al resto de la administración y al propio gobierno tuvo su ejemplificación en las primeras hojas del Registro con el Departamento Topográfico y el propio Salas:

\begin{abstract}
Por más que hemos hecho en estos últimos meses, por conseguir algunos datos del Departamento Topográfico, [...]. Nada nos ha quedado por hacer para conseguirlos, hasta ofrecernos a ir al Departamento a copiar lo que nos dieren o dictaren. Pero nos ha dicho últimamente el Sr Salas que él solo puede sacar estos datos, pues él solo sabe los libros distintos en que se encuentran, y para ello le falta tiempo. Así con esto nos creemos disculpados de no llenar debidamente esta parte tan interesante del Registro. A pesar de esto colocaremos en esta Sección (aunque algo impropiamente) ligeras noticias trasmitidas por algunos J ueces de Paz, ya sobre la topografía de sus Partidos, ya sobre la importancia rural de estos (MAESO, 1855, p. 8).
\end{abstract}

33 AHGyC, Documentos Antiguos, Departamento Topográfico, Carpeta 38, notas del 4 de diciembre de 1854

34 Maeso también hace constar la penosa tarea del reducido personal de la oficina de reiterar a distintas autoridades de la administración los "datos estadísticos correspondientes á su esfera de acción, para insertar en el Registro Estadístico". Se refiere a "esa inconmovible apatía en autoridades de un rango elevado". Maeso (1855). 
Más allá del estilo poco diplomático de Maeso, más cuando se piensa en una publicación pública, podemos ubicar el conflicto en un marco de coincidencia sobre la importancia de contar con información por parte del Estado de Buenos Aires. Los comentarios realizados por Maeso en las "Advertencias" apuntan a varias autoridades, aunque no hemos encontrado en los primeros volúmenes del Registro un comentario o queja personalizados como el realizado hacia el Presidente del Departamento Topográfico. Desconocemos cuestiones políticas o personales que pudiesen haber influido, pero sin duda, en el marco de construcción del Estado de Buenos Aires, la información sobre el territorio era relevante. Las tensiones entre Salas y las autoridades de la Mesa Estadística se concentraron en la forma de construcción del dato social y la acción propagandística que había alcanzado mayor intensidad en estos años (GONZALEZ BOLLO, 1999; OTERO, 2006).

La información obtenida de los informes o relaciones realizadas por los jueces de paz constituyó la base de la delimitación de los partidos y, al mismo tiempo, fue parte del sustento del Registro Estadístico en sus primeros tiempos. Sobre el contenido de esta información nos detendremos a continuación.

\section{Los Criterios Empleados para Establecer los Límites J urisdiccionales}

Salas le otorgaba centralidad a los "conocimientos prácticos" que los jueces de paz podían ofrecer sobre los límites de sus jurisdicciones, pese a que hemos visto, fueron logrados de diversos modos y con diferentes niveles de detalle en la información. La información presentada fue, efectivamente como lo planteaba Maeso, reproducida en el Registro Estadístico, aunque muy parcialmente (fundamentalmente aspectos descriptivos de los partidos). También, se constituyó en la base sobre la cual Salas fundamentó los criterios utilizados para proponer una nueva subdivisión de la provincia de Buenos Aires en el Registro Gráfico publicado en 1865. Sin embargo, la información no se agotó en lo antedicho y, de las variadas posibilidades que la documentación tiene, analizaremos cuáles fueron los criterios empleados por los jueces de paz para establecer los límites de las jurisdicciones, sin entrar en la información específica de cada límite. Consideramos que este aspecto de la información colabora en comprender mejor algunos cambios en las relaciones sociales y políticas de Buenos Aires a mediados del siglo XIX. 


\section{Entre los Accidentes Naturales y las Propiedades Rurales}

La jurisdicción de la provincia de Buenos Aires hacia mediados del siglo XIX era distinta a la gobernación y a la intendencia del periodo colonial. La centralidad que el territorio tenía en torno a gran parte del Río de la Plata y su vinculación con los caminos por donde transitaban personas y mercaderías por el norte de la jurisdicción vinculadas con la dinámica minera, se fue modificando y se vio desplazada por expansiones (no exentas de retrocesos) hacia el oeste y el sur agrario de su campaña. Los procesos políticos y económicos posrevolucionarios de principios del siglo XIX fueron transformando paulatinamente el territorio, que mantenía una dinámica de expansión en sus fronteras, por ejemplo durante el periodo rosista, pero también de reducción como tras la batalla de Caseros. El análisis de la dinámica poblacional refleja también estos cambios (MATEO, 2001; FRADKIN; GARAVAGLIA, 2004).

La heterogeneidad de Buenos Aires desde un punto de vista topográfico permite reconocer tres zonas internas: la costa del río de la Plata y del Océano Atlántico, la pampa ondulada con suaves lomadas que forman valles y corren caudalosos ríos y arroyos, y la pampa deprimida que se asemeja a una estepa semiárida con leves pendientes que permiten la presencia de muchas lagunas. Las diferencias se traslucen en las descripciones de los jueces de paz acerca de los límites de los partidos que tomaron como punto de referencia a arroyos, cañadas y caminos fundamentalmente durante el periodo colonial y para la zona norte de la jurisdicción. Será para la zona sur y la expansión dinamizada a partir de 1820 que este tipo de límite fue más acompañado del señalamiento de propiedades y denominaciones más abarcativas como "el Río Salado", "Río de la Plata", "el mar", y otras más genéricas vinculadas a las zonas de frontera como "la Pampa", el "desierto". Para mediados del siglo XIX, las descripciones de los jueces de paz combinan estas referencias:

Al Norte de este Pueblo es el Arroyo denominado de Suero, por unos, y de Viqueroa por otros que dista como dos leguas; que al Sur es el camino de las tropas que dista por más de media legua de este Pueblo; que al oeste esta Laguna de las Perdices [...] y al Este es la Cañada o Arroyo de la Cruz [...]" (J uez de Paz de San Andrés de Giles)

Partió con el del Salto el Camino de tropa que viene del Fortín del Salto hasta la altura de Lomas altas que para adelante tienen deslindados como lo deslinda los fondos de López Gauna y Lezica (J uez de paz de Arrecifes).

El limite divisorio entre este partido y el de Ranchos puramente convencional y de origen antiguo es un camino que viene de la Capital y al pasar por San Borombón le llaman camino del Sauce; la línea de dicho camino del Costado izquierdo pertenece a este partido y la del costado derecho a Ranchos [...]; este camino se pierde a las 
inmediaciones del establecimiento conocido por de Gándara; (J uez de Paz de Chascomús)

Linda al Norte con el de Chivilcoy, estando dividido por el Salado que por sus ondulaciones viene a dividirlo también del Partido de Chivilcoy por el Este; [...] Al oeste no tiene limites sino la Pampa desierta (Juez de Paz de Bragado)

Por un lado del Arroyo de Tapalqué rumbo al Oeste ocupado por estos indios es desierto y al Sud de este Punto siguiendo por este Arroyo también es desierto y ocupado por estos mismos indios, (Juez de Paz de Tapalqué) 35

Los criterios utilizados mantienen continuidad con los esfuerzos de precisión que los agrimensores utilizaron desde el período colonial en una topografía que no favorecía la detección de puntos relevantes para realizar las mensuras de los terrenos en estas zonas. También el Departamento Topográfico fue insistiendo en la denominación de los puntos tomados como referencias. Sin embargo, hacia 1865 la utilización de caminos, arroyos y cañadas aparece cuestionada por Salas por su cambio "constante de lugar".

A mediados del siglo XIX, los terrenos en propiedad son utilizados en la mayoría de las descripciones de los partidos (las jurisdicciones de zonas de frontera con las sociedades indígenas serían la excepción). El Juez de Paz de Bragado señalaba algunos inconvenientes en el uso de ese criterio, como "no ser perceptibles, ni están al alcance de todos, por lo que con frecuencia se ofrecen dudas". Aunque el propio juez planteara no encontrar alternativas por las características topografías del terreno. La descripción que realizó el juez de paz de Pergamino fue el caso más extremo en el uso de este criterio, ya que acude a las propiedades en todos los límites, pese a contar con arroyos relativamente importantes en esta zona de la pampa ondulada:

Lindan con el Partido de Arrecifes, y pertenecen al del Pergamino los terrenos de Don Antonio Martínez que pertenecieron al finado Don Nicolás Ferreira, los de Don Nicolás Anchorena que pertenecieron a Don José Lima Echevarría, los de Dn Francisco Alcaraz, los de Dn Mariano Alcaraz, los del finado Acosta, los de Dn Vazquez Astrada, los de Dña Desideria Casco, los de Dn Santos Barrionuevo [...]

El Juez de Paz era consciente de la decisión tomada sobre el criterio a ser utilizado, y aclaraba:

35 AHGyC, Documentos Antiguos, Departamento Topográfico, informes remitidos por los jueces de paz de San Andrés de Giles, Arrecifes, Chascomús, Bragado y Tapalqué, respectivamente, durante el año 1854 
A mi juicio, Sor Ministro, no hay otra división posible, ni mas conveniente a los propietarios que los limites de los terrenos de estos, tanto más cuanto están divididos en pequeñas fracciones, razón por la que, se ha cuidado en dar a las fracciones de terrenos, los nombres porque son más conocidos (J uez de Paz de Pergamino)

El criterio, nuevo por lo generalizado de su aplicación, fue fundamentado por la practicidad y conveniencia vinculada con las características topográficas de las distintas zonas de la jurisdicción, pese a no exteriorizarse las divisiones claramente. Sin embargo, supone para la historia de las prácticas sociales de acceso y apropiación de la tierra en la campaña de Buenos Aires una convalidación importante a favor de apellidos destacados de las élites propietarias de tierras que son los que aparecen registrados. ${ }^{36}$

Una observación interesante al respecto proviene del “ciudadano” González a quien el J uez de Paz de Villa Mercedes lo había comisionado para establecer los límites del partido, y que no era un vecinos-propietario como solían serlo ya en estos años los mismos jueces de paz. González expresaba en su informe que:

Después de seis días de trabajo he regresado a esta Villa en estado de ofrecer con seguridad una descripción de aquellos [los límites del partido, MC], los que no son en ciertos puntos otra cosa que el capricho o comodidad de los vecinos de cada uno de los partidos.

Y agregaba:

El que subscribe concluye esta nota recomendado a los VE de la comisión [en referencia al Departamento Topográfico, MC] el mejor esmero y cuidado en conservar intactos y seguros las documentos que forman su archivo; pues en ello se interesan varias consideraciones de importancia, que serán bien claras a los señores ${ }^{37}$

En la propuesta de 1865, Salas reforzará la inclusión de las propiedades en los criterios de delimitación de los partidos, expresando que: "en el proyecto que se acompaña se ha procurado no dividir ningún terreno, haciendo que los limites de estos lo sean de los Partidos;". Aún en la excepción, las propiedades fueron consideradas centrales: "excepto cuando ha habido ríos y arroyos que sirvan de deslindes a las propiedades, y que como limites naturales, han sido siempre preferidos.". ${ }^{38}$

${ }^{36}$ Ya el juez de paz de Chascomús había convocado para responder "los datos que ha obtenido este Juzgado de los propietarios y vecinos antiguos de este partido y aunque difusos ha habido que admitirlos por no tener otros mas explícitos" AHGyC, Documentos antiguos, Partido de Chascomús, nota del 11 de agosto de 1854

37 AHGyC, Documentos Antiguos, Departamento Topográfico, Juez de Paz de Villa de Mercedes, nota del 4 de diciembre de 1854

38 Salas fundamentaba: "El Departamento cree que esta [que un terreno pertenezca a más de un partido, MC] es la división mas viciosa que puede establecerse; y VE ha tenido ocasión de oír las 


\section{La Extensión de los Partidos y el Ejercicio de la Autoridad}

El "mejor ejercicio de la autoridad" sobre la población de cada la jurisdicción fue, como hemos señalado anteriormente, un criterio expresado por Salas cuando en 1853 le propuso al Ministro de Gobierno realizar la consulta a los jueces de paz para establecer los límites de los partidos. Una década después, con otro contexto en la provincia, y con las Municipalidades de Campaña funcionando, sostenía la nueva propuesta de límites de 1865 de manera análoga: "verá que se ha procurado que los Partidos sean de una extensión, que permita a las autoridades que se le den ejercer en toda ella una administración fácil.” 39

En el mismo sentido, distintos jueces de paz consideraron en 1854 la posibilidad de modificar la superficie de su partido, y la variable que fundamentó cada sugerencia estuvo vinculada, de una u otra manera, con la población. Cabe recordar que el crecimiento poblacional de Buenos Aires, ciudad y campaña, fue durante el siglo XVIII y la primera mitad del siglo XIX una característica relevante de la región. Las corrientes migratorias provenientes de zonas de lo que a partir de 1776 se constituyó como Virreinato del Rio de la Plata, fueron el pilar central del crecimiento que, además, tuvo un ritmo mayor en algunas zonas de la campaña que en la ciudad. Como hemos adelantado desde la década de 1820 se ha estimado que la población de la campaña en su conjunto, superó en cantidad a la ciudad de Buenos Aires. La antigüedad en los asentamientos en la campaña influyeron en las diferentes conformaciones sociales y económicas de Buenos Aires como territorio, pero para mediados de siglo XIX, estas diferencias parecieran haberse complejizado a partir de los procesos políticos y de movilización social de la primera mitad del siglo XIX. Para esta época, la presencia de inmigrantes trasatlánticos, perenne desde la colonia, fue generando mayor impacto.

La propuesta de Salas de 1865 tendió a la reducción de las superficies de los partidos, en sintonía con lo que venía sucediendo con las jurisdicciones civiles desde su establecimiento en el período colonial. Así fue desde las primeras divisiones en pagos caracterizados por su amplia extensión e imprecisión de sus limites-, pasando desde 1730 por la influencia de las jurisdicciones eclesiásticas conocidas como curatos, también extensas y coexistentes con las anteriores-, hasta la determinación de los primeros trece partidos en 1784 -con los Alcaldes de la Santa Hermandad como

observaciones que han hecho algunas autoridades sobre la facilidad que ella ha prestado para eludir el cumplimiento de las órdenes que dictaban, y para sustraerse a su jurisdicción lo que se conseguía con solo trasladar una población de un lado a otro, de un camino en un mismo terreno." AGN, Sala X, MGEBA, legajo 22957.

39 En 1865, Salas también incluyó las indicaciones de la Ley de 15 de septiembre de 1857, sancionada en el Gobierno de Valentín Alsina, cuyo artículo 1o establecía que "la campaña se dividiría en Departamentos, estos en Partidos que a su vez lo serían en Cuarteles y éstos en Secciones." 
autoridades a cargo-, que luego fueron aumentando en cantidad con el avance territorial y la ocupación efectiva de la frontera. En 1814 y 1821 se establecieron nuevas subdivisiones de partidos, agrupados a su vez en departamentos. Al suprimirse los cabildos y los Alcaldes de la Santa Hermandad en 1821, éstos últimos fueron reemplazados en sus funciones por los jueces de paz a cargo de los juzgados territoriales o partidos.

La reducción territorial también abarcó a las jurisdicciones eclesiásticas, ya que en 1870, al elevar el informe periódico del Poder Ejecutivo a la Honorable Asamblea Legislativa, se sintetizó la labor del Departamento Topográfico, de donde destacamos: "El Registro Grafico de las propiedades rurales de la Provincia, después de haber servido entre otros objetos administrativos, para efectuar la nueva división civil de la Campaña en J uzgados de Paz, acaba de facilitar la regulación de los limites de las Parroquias, concordándolos con los de los mencionados J uzgados. “40

Las descripciones generales sobre las jurisdicciones, como las anteriores, eclipsan en parte la complejidad de la dinámica del establecimiento de los límites. Presentamos a continuación algunos ejemplos que permiten inferirla a partir de los informes de los jueces de paz, y también evidencian la heterogeneidad y dinámica de Buenos Aires como jurisdicción.

Hacia mediados del siglo XIX, algunos jueces de paz propusieron la ampliación de los límites de sus jurisdicciones. Un ejemplo lo tenemos en los argumentos del juez de paz de Las Conchas, un partido ubicado a orillas del río Tigre frente a numerosas islas del delta al norte de Buenos Aires. A través de asentamientos espontáneos un pueblito se había consolidado durante el siglo XVIII vinculado al comercio fluvial, al contrabando y afectado por las inundaciones. En 1854, el juez de paz de las Conchas argumentaba:

Este partido será muy conveniente que fuese aumentado hasta el arroyo de Álvarez, [...], y hoy con mucho más razón desde que deben ser declarados estos distritos terrenos de pan llevar, por la causa que no hay las haciendas que en otros tiempos ha solido haber en estos terrenos de pastoreo, y que declarándose chacras, se aumentaría un gran número de familias y de hombres laboriosos y pacíficos que sería una columna fuerte para la Capital, por su aproximación a ella y las tierras y parajes a presentar para la agricultura [...] para el aumento de este partido son sobrantes del partido del Pilar, pues desde dicha línea al enunciado pueblo todavía hay distancia como mas de cinco leguas, pues al hacer el Gobierno esta nueva demarcación, nunca mejor que hoy se podría tener a la vista en favorecer a este partido tan sumamente reducido, y que de este modo dándole más amplitud daría nuevo realce a nuestro hermoso Rio del Tigre, que en

40 Informe del Poder Ejecutivo a la Honorable Asamblea Legislativa, Anexo E, 1870. 
algún tanto favorece a este Pueblo, y mucho mas desde que a solicitud de los vecinos de las Conchas, ha cedido también los de San Fernando"41

De manera similar, en una zona ubicada distante de las Conchas, al sur de Buenos Aires, el juez de paz de Dolores, un pueblo y partido formados al sur del Río Salado, frontera del avance hispanocriollo durante el siglo XVIII y primeras décadas del XIX, argumentaba:

\begin{abstract}
Siendo un inconveniente para los vecinos actuales que se hallan a corta distancia de este Pueblo y que pertenecen a otros Partidos, tener que concurrir a grandes distancias, para las situaciones y demás ocurrencias del J uzgado estando pocas cuadras de este. Pues saliendo por las Calles al Norte de este Pueblo algunas desembocan a Campo de Peredo Partido del Tordillo, [...]
\end{abstract}

La propuesta de nuevos límites del J uez de Paz de Dolores incluía a las Islas o Montes del Tordillo, pertenecientes a la jurisdicción homónima. Estas eran aun a mediados del siglo XIX:

Parte muy esencial para esta población que de ella se surte de la leña y carbón y según tradición de los antiguos pobladores tiene este Pueblo [...] que hasta hoy es propiedad del Estado pongan según se les hubo de mensurarse esos terreno por D. Santiago Tobal y los vecinos de este Pueblo se presentaron al Gobierno consiguiendo que pagando ellos los trabajos emprendidos por el agrimensor quedase como de propiedad del Estado como hasta hoy existe.

Otra razón para que forme parte del Partido de Dolores es que la inmediación de la autoridad que reside en este Pueblo tendría más influencia que la que hoy tiene por el Juez de Paz de ese Pueblo (Tordillo, MC), que por la distancia no puede hacer tan eficaz la vigilancia precisa en las gentes nuevas que se abrigan allí y asolan los alrededores viviendo de las haciendas afines estos días ha tenido este J uzgado diferentes quejas de esa partes inmediatas a los montes."

En zonas de conformación distintas, y distantes entre ellas, los jueces de paz señalan aspectos en común a tener en cuenta para mejorar la situación de sus partidos: recursos para la población, o para atraerla; la ubicación del pueblo (y paulatinamente de la autoridad) en cada jurisdicción; el asentamiento de "familias y hombres laboriosos", y lograr vigilar a "gentes nuevas" de los montes.

En otras oportunidades, algunos jueces de paz coincidieron en la posibilidad de dividir los partidos para equilibrar la superficie con la población. En el caso de Ensenada y Magdalena, cada juez lo proponía de manera cruzada avanzando primero sobre el partido lindero. El juez de Paz de Magdalena, un partido que durante el

${ }^{41}$ AHGyC, Documentos antiguos, Partido de las Conchas. “ 
periodo colonial se destacó por su orientación ganadera, su extensión y relativa baja densidad de población, consideraba:

[...] muy conveniente formar dos Partido de lo que hoy reconoce la práctica por éste, agregándole el Cuartel que hoy se conoce por el 3 o de la Ensenada; y de este modo quedarían divididos por limites naturales, con una figura regular en longitud y ancho, con una extensión de leguas cuadradas y una población casi igual a uno y otro.

El cálculo realizado dejaba un partido con unos 1900 habitantes, y remarcaba la pronta posibilidad de conformar un pueblo en el mismo.

[...] con un punto muy poblado, cual es el vecindario del Pescado con terrenos adyacentes, que ya no sirven para cría de ganado, sino para de pan llevar, que en un tiempo corto sería un pueblo, pues tiene la ventaja a más de su crecido vecindario de quedar en la Carrera del de la Ensenada al de Sta. Ma. Magdalena, y a una distancia como de cinco leguas de uno y otro [...].

El otro partido comprendería "el Pueblo de Sta. Ma. Magdalena con una población de 2000 almas"

El juez de paz de Ensenada, por su parte, fundamentaba la incorporación de territorio del partido de Magdalena "por no haber hacia esta parte ningún límite natural que adoptar y por estar el Pueblo de la Ensenada en una de los ángulos del territorio, siempre habría ventajas en que el ángulo opuesto no se alejara tanto del Pueblo, cabeza de Partido." En su propuesta, quedarían dos partidos, uno con 2700 habitantes y el otro que "en proporción a su tamaño la diferencia vendría a ser poco sensible"42

También se presentaron fundamentaciones para modificar los limites de los partidos basadas en cuestiones específicamente políticas. Tal el caso del partido de Lobos, donde la ampliación planteada por el Juez de Paz correspondió a la reivindicación de la estancia "Altos verdes", que:

[...] debiendo pertenecer como ha pertenecido a este Partido por limites naturales, designados por los expresados arroyos esta agregado indebidamente al Partido del Monte, desde la Administración de Dn Juan M. Rosas" [...] "por razón natural, y por que el área que comprende el Partido sea mas regular;"

Una clara propuesta de división territorial fue la del juez de paz de Tandil, en la frontera sur, quien proponía fuesen tres los partidos: Chapaleufú con "800 y tantos

42 AHGy C, Documentos antiguos, partido de Magdalena y Ensenada, respectivamente 
habitantes"; Napaleufú (o Arroyo Chico o Cherforó) con una población de 1000 habitantes, y Tandil, con su pueblo como centro del nuevo partido y unos 1100 pobladores.

Por último otro tipo de argumentación sobre la división de los partidos fue el esgrimido por el juez de Paz de la Matanza:

[...] es inconveniente alguna subdivisión en un Partido, si ella debe hacerse desmembrando un territorio para agregarlo a otro, por que mas o menos cada un habitante posee el espíritu de localidad y se enorgullece de pertenecer a aquel en que nació o tiene su fortuna y esto sucede en punto mayor de nación a nación, como de un barrio a otro de una ciudad" $[. . .]^{43}$

Y aclaraba que si era necesario efectuarlo, "menos fuerte será la ejecución si se la practica por las municipalidades". J ustamente es en 1854 cuando se sanciona la Ley de las Municipalidades, que será reglamentada en 1856 y puesta en práctica paulatinamente según los casos.

Por último, algunos jueces de paz directamente sostuvieron la necesidad de aumentar la población de su jurisdicción, sin considerar relevante el tema de la extensión. La situación de la frontera más distante de la ciudad de Buenos Aires brinda varios ejemplos: tras considerar los puñados de habitantes que se encontraban en el Río Colorado y el Sauce Chico, el J uez de Paz de Bahía Blanca estima un total de 1500 pobladores en el distrito "según ha quedado reducida por las invasiones de los indios". Agregando que:

Están concentradas bajo las fuerza de la Fortalezas quedando también campos para el procreo de ganados y agricultura, desiertos en toda su extensión. El infrascrito cree que tan corta población, el suelo como está distribuido y los limites tan bien marcados de los territorios, no dan lugar al menos por ahora a imaginar subdivisión ${ }^{4}$

Sin embargo, así como se señalaron los ataques indígenas en algunas descripciones de los jueces de paz, en otros casos como en Tapalqué se hizo referencia a los "indios mansos" y al interesante proceso de conformación del juzgado:

En el año 33 por disposición del gobierno se situaron los indios mansos en este Punto a una y otra banda del Arroyo Tapalqué en donde se estableció una comandancia militar y se permitió establecer

43 AHGy C, Documentos antiguos, partido de la Matanza

${ }^{44}$ Al describir los limites, el Juez de Paz de Bahía Blanca señalaba "los campos de Manuel Mapú y Salinas Grandes hoy posesión de los Indios, carece el territorio del partido en limites naturales bien marcados. Es por consiguiente la configuración un paralelogramo cuyos lados más cortos son el mar y su opuesto." AHGyC, Documentos antiguos, Partido de Bahía Blanca. 
algunos negociantes que hacían y hacen el negocio de cambio con dichos indios hasta hoy; que sucesivamente fueron poblando algunas chacras y estancias, las que existen en corto número. En el año 48 dispuso el gobierno de entonces que dicha comandancia se denominase J uzgado de Paz, en este estado el nuevo Juez acordó con el Juez de Paz de Azul lindero, que los fondos de las suertes de estancia del arroyo Azul se conocieran por limites[... $]^{45}$

\section{Conclusiones}

Los procesos de articulación y negociación no fueron las únicas formas utilizadas por los grupos dirigentes para encontrar soluciones a los desafíos de la construcción de un nuevo proyecto de Estado tras Caseros, pero sí, posiblemente, los que resultaron más estables. Analizados a partir de la construcción del territorio de la provincia, encontramos que éstos no fueron simples ni veloces.

Nos hemos centrado en dos experiencias que se desarrollaron en el incipiente y convulsionado Estado de Buenos Aires. Por un lado, la dinámica generada a partir del impulso del Departamento Topográfico de Buenos Aires para obtener información sobre los límites de los partidos, que fomentó la realización de informes por parte de los jueces de paz sobre sus jurisdicciones. Por el otro, las solicitudes realizadas por la Mesa Estadística al mismo Departamento con el objetivo de publicar información sobre el territorio de manera periódica en el Registro Estadístico.

Las construcciones del dato social tanto a nivel local como dentro de la administración provincial no tuvieron resoluciones únicas. Sin embargo, pudimos señalar algunas características del proceso.

Una particularidad fue la carencia de información precisa sobre los límites de los partidos por parte de la administración provincial y de los jueces de paz a mediados del siglo XIX, y la valoración otorgada al "conocimiento práctico" como la base para obtenerla. La construcción del territorio apareció descentrada de la administración provincial que si bien lo impulsó, resultó deudora en gran medida de los poderes locales que obtuvieron y delimitaron la información. Específicamente, los informes de los jueces de paz fueron utilizados, parcialmente, para el Registro Estadístico y en nombre del Departamento Topográfico, Saturnino Salas los consideró para sustentar los criterios de su propuesta de límites que acompañó el Registro Gráfico de 1865.

La construcción por parte de la administración de instrumentos vinculados al territorio y considerados necesarios para gobernar fue dilatada. Además de la coyuntura política compleja, influyeron las diferentes reacciones o posibilidades de los jueces de paz para realizar sus informes. Se pusieron en evidencia las limitaciones de la

45 AHGyC, Documentos antiguos, Partido de Tapalqué. 
administración -en proceso de reorganización- para la obtención de información, generadoras de pedidos al ministro de gobierno de "ordenar" el cumplimiento o generar "sistemas coercitivos" tanto por parte de Salas como de Maeso. La importancia de los procesos de acumulación de información y contrastación de datos por parte del Departamento Topográfico y la administración en general resultó determinante para comprender la dilatación de los logros. En paralelo, y en tensión, la importancia de la difusión por parte del gobierno que hizo mas ambiciosa la función propagandista del Estado, favorecida por el uso más generalizado de la imprenta.

Los criterios utilizados por los jueces de paz para establecer los límites de sus jurisdicciones mantuvieron, en parte, continuidad con las prácticas de los agrimensores que recuperaban las características topográficas de la pampa ondulada y deprimida, es decir arroyos, cañadas, lagunas, y también caminos. Sin embargo, a mediados del siglo XIX los jueces de paz incorporan con mayor asiduidad el uso de las propiedades como criterio para establecer los limites de los partidos. Más allá de alguna voz que recomendó esmero en la consulta de la documentación y el cuidado de los archivos, y catalogaba algunos limites establecidos como comodidad de los vecinos de cada partido, fue también incorporado por Salas en su propuesta de 1865. El criterio de delimitación a partir de las propiedades aparecía fundado en la practicidad y conveniencia, pero supuso la aceptación de los límites de las propiedades y una convalidación importante a favor de apellidos destacados de la elite regional y locales.

La búsqueda del mejor ejercicio de la autoridad sobre la población de cada jurisdicción fue el criterio sostenido por Salas cuando en 1853 le propuso al ministro de gobierno realizar la consulta a los jueces de paz, y fue el que reitero en 1865. En el mismo sentido, distintos jueces de paz consideraron la posibilidad de modificar la superficie de su partido, siendo la variable población la que fundamentó cada sugerencia, de una u otra manera. Esta finalidad fue compartida en la provincia, más allá de las notorias diferencias de densidad poblacional, y aun en una campaña en crecimiento.

El conocimiento del territorio como instrumento para un mejor accionar de los gobiernos no surgió, hemos visto, por primera vez a mediados del siglo XIX. Una nueva combinación de situaciones permitió actualizar esta iniciativa. Hemos argumentado que los procesos de articulación para consensuar límites jurisdiccionales que permitiesen un mejor control del territorio y la población implicaron hacia mediados del siglo XIX, la aceptación de distintas relaciones sociales y políticas por parte de las autoridades locales y provinciales. El proceso estudiado marcaría un intento por establecer la centralidad de los partidos, acoplando a ellos a las jurisdicciones 
eclesiásticas, posiblemente a la luz del establecimiento de las municipalidades y demás cambios políticos y sociales que el Estado de Buenos Aires se proponía.

\section{Referências}

BANZATO, G. Ocupación y acceso a la propiedad legal de la tierra en la región nordeste del río Salado : chascomús, ranchos y monte, 1780-1880. 2002. Tesis (Doctorado en Historia) - Facultad de Humanidades y Ciencias de la Educación. Universidad Nacional de La Plata - UNLP, La Plata.

BONAUDO, M. Liberalismo, Estado y orden burgués (1852-1880). 2. ed. Buenos Aires: Sudamericana, 2007.

BUENOS AIRES. Provincia. Dirección de Geodesia y Catastro. Manual de disposiciones usuales para la Dirección de Geodesia. Antecedentes de la Repartición y Archivo, La Plata: Talleres Impresiones Oficiales, 1947. Tomo I, p. 3-4.

CANEDO, M. Mucho más que una cuestión de medidas. Las comisiones para el arreglo de los pueblos del Estado de Buenos Aires. Pergamino, Arrecifes, San Pedro, 18541856. En: GARAVAGLIA, J . C.; GAUTREAU, P. (Ed.). Mensurar la tierra, controlar el territorio América Latina, siglos XVIII-XIX. Rosario: Prohistoria, 2011. p. 259-295.

ESTEBAN, F. El Departamento Topográfico de la Provincia de Buenos Aires (Actual Dirección de Geodesia), Buenos Aires, 1962.

FRADKIN, R.; GARAVAGLIA, J . C. (Ed.). En busca de un tiempo perdido. La economía de Buenos Aires en el país de la abundancia, 1750-1865. Buenos Aires: Prometeo, 2004.

GAUTREAU, P.; BOULLIER, J .; CUÉNOT, J . F. Catastro, construcción del Estado e institucionalización administrativos en la provincia de Buenos Aires y Uruguay (18201870): enfoques geohistóricos. En: GARAVAGLIA, J . C.; GAUTREAU, P. (Ed.). Mensurar la tierra, controlar el territorio América Latina, siglos XVIII-XIX. Rosario: Prohistoria, 2011. p. 97-150.

GAUTREAU, P.; GARAVAGLIA, J . C. Inventando un nuevo saber estatal sobre el territorio: la definición de prácticas, comportamientos y agentes en las instituciones topográficas de Buenos Aires, 1824-1864. , GARAVAGLIA, J . C.; GAUTREAU, P. (Ed.). Mensurar la tierra, controlar el territorio América Latina, siglos XVIII-XIX. Rosario: Prohistoria, 2011. p. 63-96.

GELMAN, J . Crisis y reconstrucción del orden en la campaña de Buenos Aires. Estado y sociedad en la primera mitad del siglo XIX. Boletín del Instituto de Historia Argentina y Americana ‘Dr. Emilio Ravignani, Buenos Aires, n. 21, 2000.

GONZALEZ BOLLO, H. Génesis y funciones de los manuales estadísticos y geográficos, 1852-1876. Anuario del IEHS, Tandil, n. 14, 1999.

HALPERIN DONGHI, T. Proyecto y construcción de una nación (1846-1880). Buenos Aires, Ariel, 1995.

LETTIERI, A. La construcción de la República de la opinión. Buenos aires frente al interior en la década de 1850. Buenos Aires, Prometeo, 2006. 
MAESO, J . Registro estadístico del Estado de Buenos Aires correspondiente al

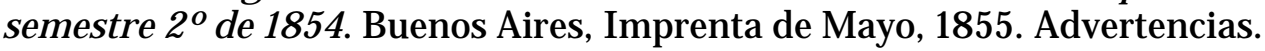

MATEO, J . Poblacion, parentesco y red social en la frontera. Lobos en el siglo XIX. Mar del Plata: GIHRR-UNMDP, 2001.

OTERO, H. Estadistica y Nación: una historia conceptual del pensamiento censal de la Argentina Moderna, 1869-1914. Buenos Aires: Prometeo, 2006.

PRO, J . Mensuras, catastro y construcción estatal. En: GARAVAGLIA, J . C.; GAUTREAU, P. (Ed.). Mensurar la tierra, controlar el territorio América Latina, siglos XVIII-XIX. Rosario: Prohistoria, 2011. p. 97-150.

VALENCIA, M. Tierras publicas, tierras privadas. Buenos Aires 1852-1876. La Plata: UNLP, 2005.

ZEBERIO, O. El liberalismo y los derechos de propiedad en Argentina. Controversias jurídicas y proyectos políticos en la etapa codificadora. En: BLANCO, G.; BANZATO, G. (Comp.). La cuestión de la tierra pública en Argentina: a 90 años de la obra de Miguel Ángel Cárcano. Rosario: Prohistoria, 2009. p. 35-56.

ZINNY, A. Historia de los Gobernadores de las Provincias Argentinas. Buenos Aires: Hyspamerica, 1987. Tomo 1.

Colaboración recibida en 04/ 02/2011 y aprobado en 18/08/2011. 
Mariana Canedo

Construyendo el territorio tras Caseros: los jueces de paz y la administración pública en la formación ... 